\title{
Étude génétique de la réaction à la pourriture brune des cabosses chez des cacaoyers (Theobroma cacao L) issus d'un plan de croisements diallèle
}

\author{
D Berry ${ }^{1}, \mathrm{C}$ Cilas ${ }^{2 *}$ \\ ${ }^{1}$ IRA/CIRAD-CP phytopathologie, centre IRA de Nkolbisson, BP 2067, Yaoundé, Cameroun \\ ${ }^{2}$ CIRAD-CP biométrie, BP 5035, F34032 Montpellier, France
}

(Reçu le 5 septembre 1994 ; accepté le 21 décembre 1994)

\begin{abstract}
Résumé - La production et la pourriture brune des cabosses du cacaoyer due à Phytophthora megakarya ont été étudiées durant 3 années consécutives dans un essai diallèle complet $6 \times 6$, sans les autofécondations, planté en 1974 dans la station de Barombi-Kang (Cameroun). Les aptitudes générales à la combinaison (AGC) constituent les seules sources de variation significatives pour la production réelle et le taux de pourriture ; la supériorité du géniteur UPA 134 est confirmée. Des modèles logistiques permettent de paramétrer la dynamique de l'épidémie. Une analyse diallèle des paramètres définis selon ces modèles est proposée. Les vitesses maximales d'évolution de la maladie sont héritables et conditionnent les taux de pourriture finaux. Le classement du matériel végétal étudié diffère de celui obtenu par tests d'inoculation artificielle sur cabosses. La répartition verticale des fruits dans les arbres et la charge en cabosses des différents croisements ne permettent pas d'expliquer simplement cette divergence. Une hypothèse est formulée pour expliquer les différences de classements entre les 2 modes d'évaluation du matériel végétal : les clones à cycle de fructification court apparaissent moins sensibles au champ que lors des tests d'inoculations artificielles car l'exposition de leurs cabosses au pathogène dure en moyenne moins longtemps.
\end{abstract}

cacaoyer / pourriture brune /Phytophthora megakarya / facteurs de résistance / diallèle

Summary - Genetic study of cocoa (Theobroma cacao L) behaviour towards black pod disease caused by Phytophthora megakarya. The relationship between yield and black pod disease of cocoa caused by Phytophthora megakarya has been studied over 3 years in a diallel $6 \times 6$ trial set in Barombi-Kang experimental station (Cameroon) in 1974. The general combining abilities were the only significant factors on effective yield and black pod losses. The superiority of the parent UPA 134 was confirmed. Logistic models were used to define the parameters of the epidemic dynamics. A diallel analysis of these parameters was suggested. The maximum speed of disease propagation was heritable and the black pod losses were dependent on this parameter. The classification of the clones was different from that obtained with pod artificial inoculation tests. The vertical pod distribution in the trees and pod load of the different crosses could not explain this divergence. A hypothesis was proposed to explain the clone classification differences between these 2 evaluation modes: clones with a short pod cycle seem to be less susceptible in the field than during artificial pod inoculations because the pathogenic exposure of their pods was shorter.

cocoa / black pod disease / Phytophthora megakarya / resistance factor / diallel

* Correspondance et tirés à part. 


\section{INTRODUCTION}

La pourriture brune des cabosses est l'une des maladies les plus répandues chez l'espèce Theobroma cacao $\mathrm{L}$; elle provoque des pertes de production importantes, estimées à près de $30 \%$ à l'échelle mondiale (Lass, 1985). Les fèves issues de cabosses atteintes de pourriture brune sont en effet impropres à la consommation et sont le plus souvent détruites.

Au Cameroun, cette maladie revêt une importance particulière. Des taux d'attaque de $50 \%$ de la production sont couramment observés chaque année; dans des zones où les conditions écologiques sont très favorables au développement de la maladie, les pertes peuvent atteindre $80 \%$ de la récolte (Despréaux et al, 1988).

Cette maladie est due à un champignon appartenant au genre Phytophthora. Au Cameroun, des études récentes ont mis en évidence la présence d'une seule espèce : Phytophthora megakarya (Nyasse, 1992) ; celleci est considérée comme l'espèce la plus agressive (Brasier et Griffin, 1979).

Pour contrôler cette maladie, la lutte chimique est souvent utilisée par les producteurs. Malgré de récents travaux montrant qu'une diminution de la charge de travail est possible avec la mise en œuvre d'un protocole d'intervention à 4 applications annuelles de fongicides (Berry et Tafforeau, non publié), la lutte chimique reste coûteuse, plus particulièrement dans le contexte actuel de baisse du prix du cacao sur le marché mondial.

La sélection de cacaoyers présentant une moindre sensibilité à la pourriture brune des cabosses est donc actuellement un axe de recherche prioritaire. Jusqu'à présent, malgré de nombreux travaux (Thorold, 1953 ; Tarjot, 1969 ; Blaha et Lotodé, 1976), la mise en évidence de cacaoyers présentant un haut niveau de résistance au champ est restée vaine. De nombreux auteurs suggèrent que les différences de réaction à Phytophthora $s p$ relèvent d'une résistance partielle, probablement polygénique (Partiot, 1975 ; Blaha et Lotodé, 1977).

Différentes méthodes d'évaluation du matériel végétal ont été éprouvées (Blaha, 1974) : l'observation du comportement au champ en condition naturelle d'infection et les tests d'inoculation artificielle sur cabosses demeurent les 2 principales méthodes retenues. Cette dernière méthode a été utilisée pour comparer la sensibilité de différents clones par Blaha et Lotodé (1976) ; ces auteurs ont décomposé la résistance au Phytophthora $s p$ en 2 principales composantes: la résistance à la pénétration et à la progression du parasite dans les tissus. À partir du classement des clones obtenu par ces tests, 6 parents ont été choisis dans une gamme très large de réaction au pathogène pour réaliser un essai diallèle au Cameroun.

Une première étude du comportement de cet essai vis-à-vis de la pourriture brune des cabosses, en conditions naturelles d'infection (Despréaux et al, 1989), a révélé l'existence d'un caractère de résistance partielle au champ génétiquement transmissible.

Afin d'identifier différents facteurs pouvant intervenir dans l'expression de ce caractère, des observations sur les niveaux et la dynamique de la pourriture brune, ainsi que sur la production et sa répartition, ont été effectuées durant 3 années consécutives dans un essai diallèle de la station IRA (Institut de recherche agronomique du Cameroun) de Barombi-Kang.

\section{MATÉRIEL ET MÉTHODES}

\section{Matériel observé}

Les cacaoyers observés sont issus d'un plan diallèle complet $6 \times 6$ (sans les autofécondations). Cet essai, planté à la station de Barombi-Kang en 1974, est constitué de 6 blocs. Dans chaque bloc, 12 arbres par famille sont disposés en randomisation totale, suivant une densité de 1330 pieds/ha.

Les 6 géniteurs sont, par ordre de sensibilité décroissante à la pourriture brune : SNK 10, UPA 134, IMC 67, ICS 95, SNK 413 et ICS 84 (Blaha et Lotodé, 1976).

SNK 10 et SNK 413 sont des Trinitario locaux, ICS 95 et ICS 84 sont des Trinitario de Trinidad sélectionnés par l'ICTA, UPA 134 est une descendance de Forastero Haut Amazonien en provenance du Ghana (WACRI) et IMC 67 est un Haut Amazonien collecté à lquitos (Pérou).

\section{Méthodes}

\section{Observations}

Chaque arbre en essai a été observé pendant la période de fructification (mai-novembre), durant les 3 années : 1988, 1989 et 1990.

Les cabosses pourries (atteintes de pourriture brune), wiltées (dessèchement précoce de nature physiologique), endommagées par les rongeurs et mûres 
saines ont été dénombrées chaque semaine. Lors de ces comptages, une récolte sanitaire est effectuée : toutes les cabosses, à l'exception des saines non mûres, sont prélevées. Les données sont enregistrées par famille et par bloc. La situation topographique des différentes cabosses dénombrées est également enregistrée suivant les 2 principaux niveaux de fructification : tronc et branches.

Les pertes dues à la pourriture brune sont estimées par rapport à la production potentielle (hors cabosses rongées) selon la formule:

\section{$\sum$ cabosses pourries}

taux de pourriture $=$

$\sum$ cabosses pourries

$+\sum$ cabosses mûres

$+\sum$ saines au dernier comptage

Les données, enregistrées par semaine, permettent également de calculer un pourcentage cumulé de pourriture brune et d'en suivre l'évolution au cours du temps.

\section{Analyses}

L'analyse diallèle du dispositif en bloc est réalisée selon la méthode de Griffing (1956), suivant le modèle à effets fixes :

$$
P_{i j k}=\mu+g_{i}+g_{j}+m_{i}-m_{j}+s_{i j}+r_{i j}+E_{i j k}
$$

avec $g_{i}$ : aptitude générale à la combinaison du géniteur $i ; m_{i}$ : effet général de réciprocité (ou effet maternel) du géniteur $i ; s_{i j}$ : aptitude spécifique à la combinaison du couple $i j ; r_{i j}$ : effet spécifique de réciprocité du couple ij.

Les différents caractères étudiés sont :

- la production potentielle (nombre total de cabosses formées);

- la production réelle (nombre de cabosses mûres récoltées) ;

- le taux de pourriture annuel ;

- les taux de pourriture pour les cabosses du tronc et des branches ;

- la répartition verticale de la production (tronc, branches), estimée par le rapport : nombre total de cabosses produites dans les branches / nombre total de cabosses produites dans l'arbre entier.

L'étude dynamique de la progression de la maladie dans le temps est réalisée sur l'ensemble des arbres du diallèle pour chacune des 3 années : une régression non linéaire est effectuée sur les pourcentages cumulés de pourriture brune en fonction du temps, selon le modèle logistique, qui s'est avéré le plus adapté :

$$
Y=P_{1} /\left(1+\exp \left(P_{2}-P_{3} \cdot t\right)\right)
$$

avec $Y$ : pourcentage cumulé de pourriture brune; $t$ : nombre de semaines depuis le début des fructifications.
Les paramètres $P_{1}, P_{2}$ et $P_{3}$ ont été estimés par la méthode de Gauss-Newton (Hartley, 1961).

Sur les données de la première année (1988), des ajustements logistiques ont été réalisés pour chacune des 30 familles sur l'ensemble de la parcelle. En l'absence d'effet résiduel estimé, un modèle d'analyse diallèle est proposé pour comparer certains paramètres du modèle : les AGC (aptitudes générales à la combinaison) sont testées par rapport aux ASC (aptitudes spécifiques à la combinaison) et les EGR (effets généraux de réciprocité ou effets maternels) sont testés par rapport aux ESR (effets spécifiques de réciprocité) (Dattée, 1972).

Enfin, les corrélations phénotypiques, génétiques, et environnementales sont calculées entre les taux de pourriture et les variables de production potentielle et de répartition verticale des cabosses.

\section{RÉSULTATS}

\section{Analyse diallèle des principaux caractères}

Quatorze années après la mise en place de l'essai diallèle, 1526 arbres survivants sont dénombrés ; l'effectif initial était de 2160 arbres (72 pieds par famille).

Lors de ces 3 années d'observation, un très faible taux de cabosses wiltées a été enregistré (environ $10 \%$ ), alors que des taux de $50 \%$ sont fréquemment rencontrés dans d'autres parcelles de la station. Les pertes de cabosses dues aux rongeurs ont en revanche été très importantes, avec des taux moyens de $20 \%$ à $50 \%$.

L'étude de la production potentielle ne permet de mettre en évidence un effet famille qu'en 1989 , année pour laquelle cette production était la plus faible (tableau I). En revanche, l'effet famille est significatif chaque année sur la production réelle. Un effet important des familles est observé sur les taux de pourriture, excepté pour 1990 , année pour laquelle les taux de pourriture ont été exceptionnellement forts (tableau I), avec un tassement des valeurs vers la borne supérieure $(100 \%)$. Les effets blocs sont très significatifs pour l'ensemble des caractères et des années, ce qui implique que les blocs contrôlent des effets environnementaux importants.

Les analyses diallèles sont présentées dans le tableau II. Les effets génétiques sont peu importants pour la production potentielle; ce caractère paraît donc assez peu héritable. En revanche, l'effet des AGC, seule source de variation significative, est très important pour les caractères de production réelle et de taux de pourriture ; une 
héritabilité de type essentiellement additif semble donc régir la transmission de ces caractères, ce qui confirme des études antérieures (Despréaux et al, 1989 ; Tan et al, 1990).

Les estimations des AGC par géniteur, ainsi que les tests de comparaison multiple de Newman et Keuls, sont présentés pour les variables de production ayant des effets significatifs pour cette source de variation (tableau III). Des groupes homogènes se dégagent assez nettement pour les productions réelles, avec, notam- ment, la supériorité constante du géniteur UPA 134.

Ces comparaisons sont ensuite effectuées sur les taux de pourriture (tableau IV). La supériorité du géniteur UPA 134 est également mise en évidence pour ce caractère. II est à noter que ce classement, obtenu sur les AGC, est à peu près identique à celui observé sur les valeurs propres des clones estimées dans un essai clonal situé dans la même station expérimentale (Berry et Cilas, 1994). En revanche, ces classements sont

Tableau I. Effet des facteurs famille et bloc sur les caractères étudiés. Moyennes, valeurs des F (test de Fisher) et probabilités associées.

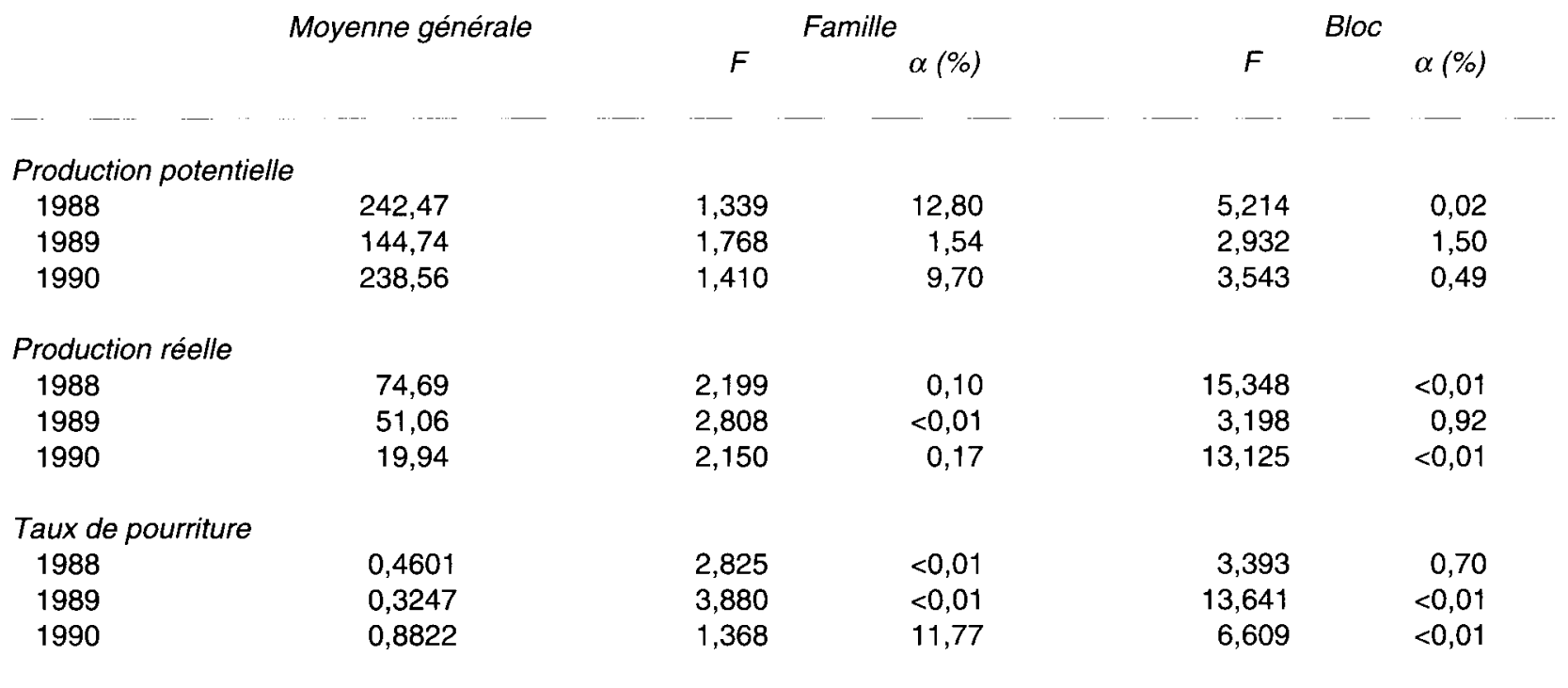

Tableau II. Valeurs des F du test de Fisher pour les différents effets étudiés dans l'analyse diallèle du dispositif en blocs.

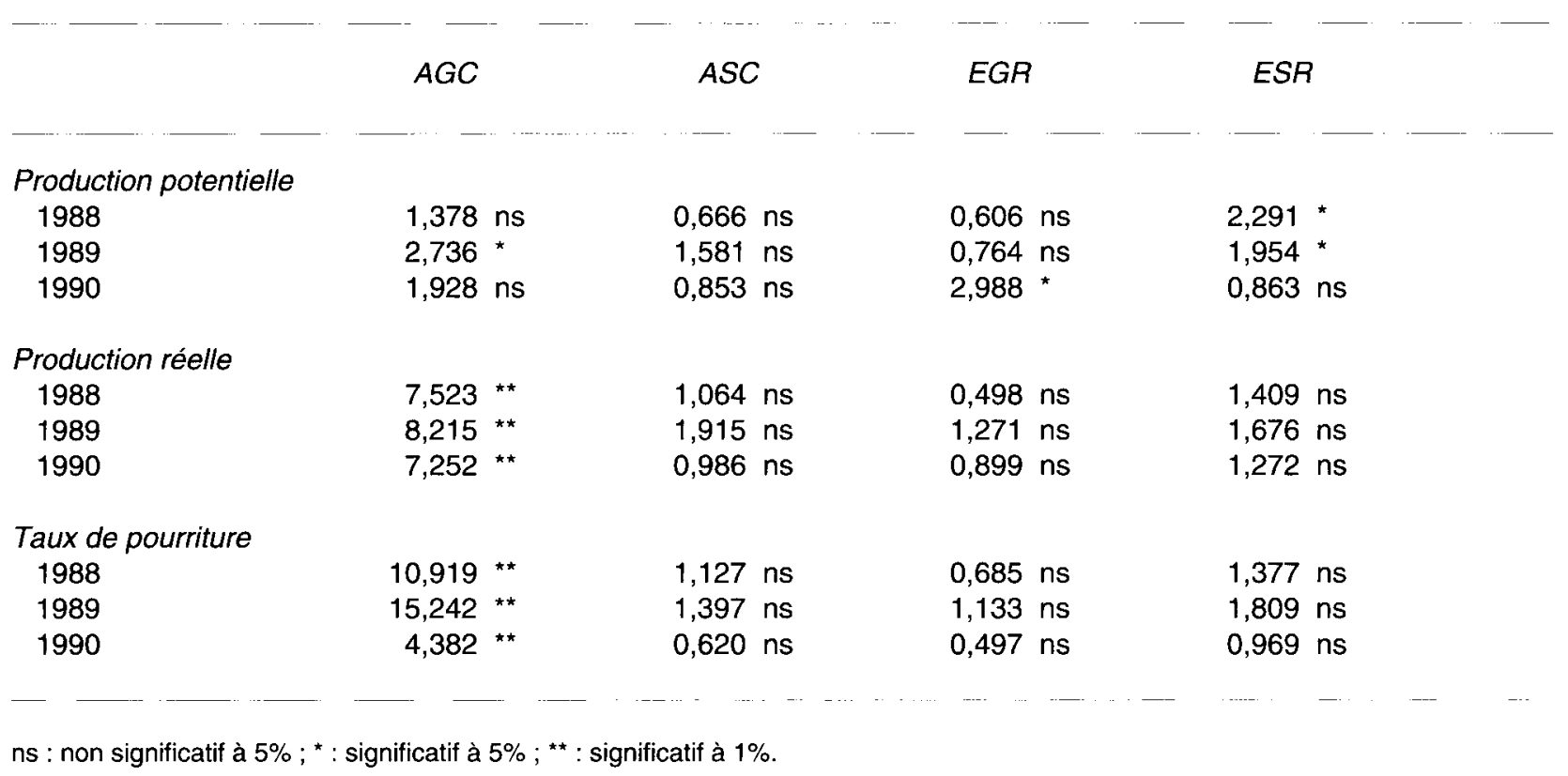


Tableau III. Valeurs estimées des AGC par année et par géniteur $\left(\hat{g}_{i}\right)$ et tests de comparaison multiple de Newman et Keuls à $5 \%(\mathrm{~N}$ et $\mathrm{K})$, pour les caractères de productions potentielle et réelle.

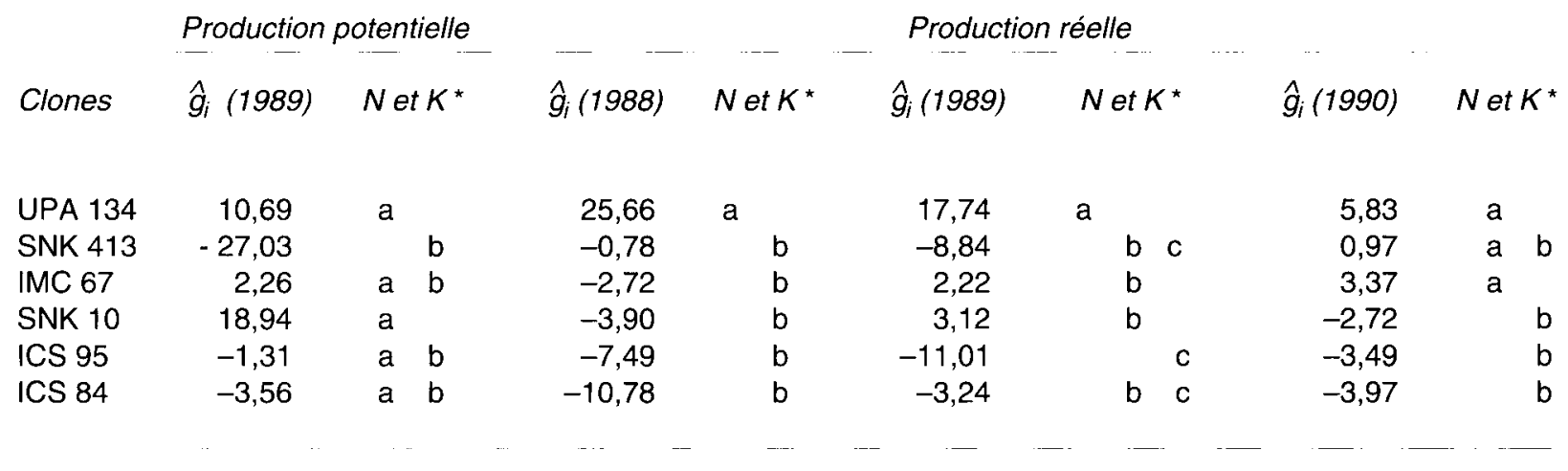

* Les clones avec la même lettre ne sont pas différents au seuil de $5 \%$.

Tableau IV. Valeurs estimées des AGC par année et par géniteur $\left(\hat{g}_{i}\right)$ et tests de comparaison multiple de Newman et Keuls à $5 \%$ ( $N$ et $K)$, pour les taux de pourriture (en \%)

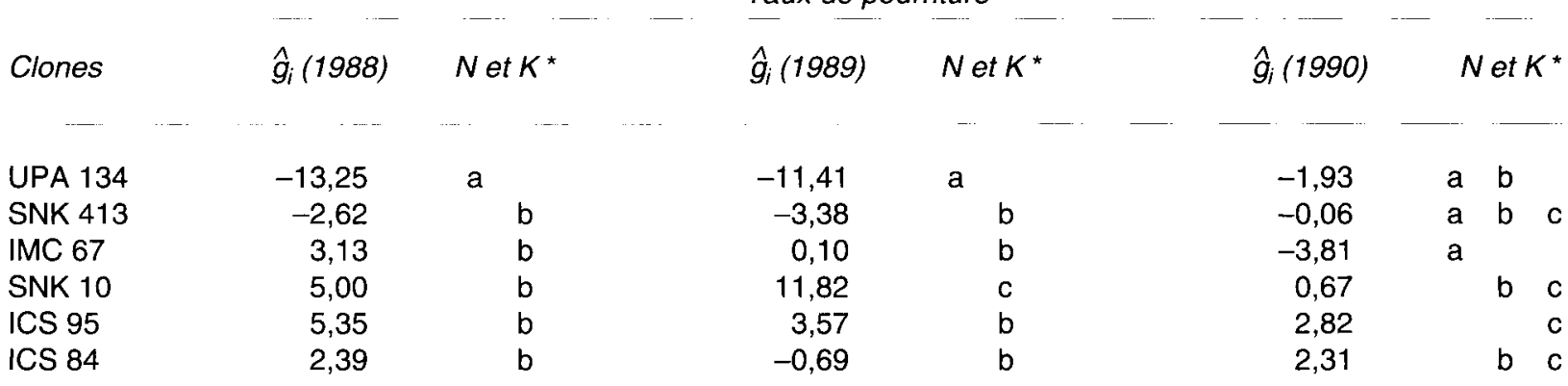

* Les clones avec la même lettre ne sont pas différents au seuil de 5\%.

différents de ceux observés par tests d'inoculation sur cabosses (Blaha et Lotodé, 1976). Un résumé des différentes échelles de sensibilité en fonction du mode d'évaluation est présenté dans le tableau V.

Des éléments pour expliquer ces divergences de classement sont apportés dans la suite de ce travail.

\section{Dynamique de l'épidémie}

Pour chacune des 3 années d'observation, les ajustements logistiques sont satisfaisants avec des $R^{2}$ variant de 0,995 à 0,999 (figs 1,2 et 3 ) : ils permettent de rendre compte de l'évolution de la maladie au cours des campagnes de fructification. D'autres modèles ont également été testés

Tableau V. Effet de la méthode de test de la résistance sur le classement des clones utilisés.

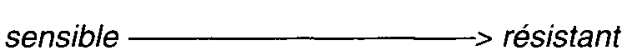

$\begin{array}{lllllll}\text { Test d'inoculation artificielle sur cabosses * }^{*} & \text { SNK 10 } & \text { UPA 134 } & \text { IMC 67 } & \text { ICS 95 } & \text { SNK 413 } & \text { ICS 84 } \\ \text { Valeurs propres des clones (essai clonal) }^{\star *} & \text { SNK 10 } & \text { ICS 95 } & \text { IMC 67 } & \text { ICS 84 } & \text { UPA 134 } & \text { SNK 413 } \\ \text { AGC globale des géniteurs (diallèle) }{ }^{\star \star \star} & \text { SNK 10 } & \text { ICS 95 } & \text { IMC 67 } & \text { ICS 84 } & \text { SNK 413 } & \text { UPA 134 }\end{array}$

* Blaha et Lotodé, 1976 ; *^ Berry et Cilas, $1993 ;{ }^{\star \star \star}$ résultats de cette étude (cumul des 3 années) et Despréaux et al, 1989. 
Fig 1. Ajustement du pourcentage cumulé de pourriture brune à une loi logistique (année 1988) $\left(R^{2}=0,999\right)$.
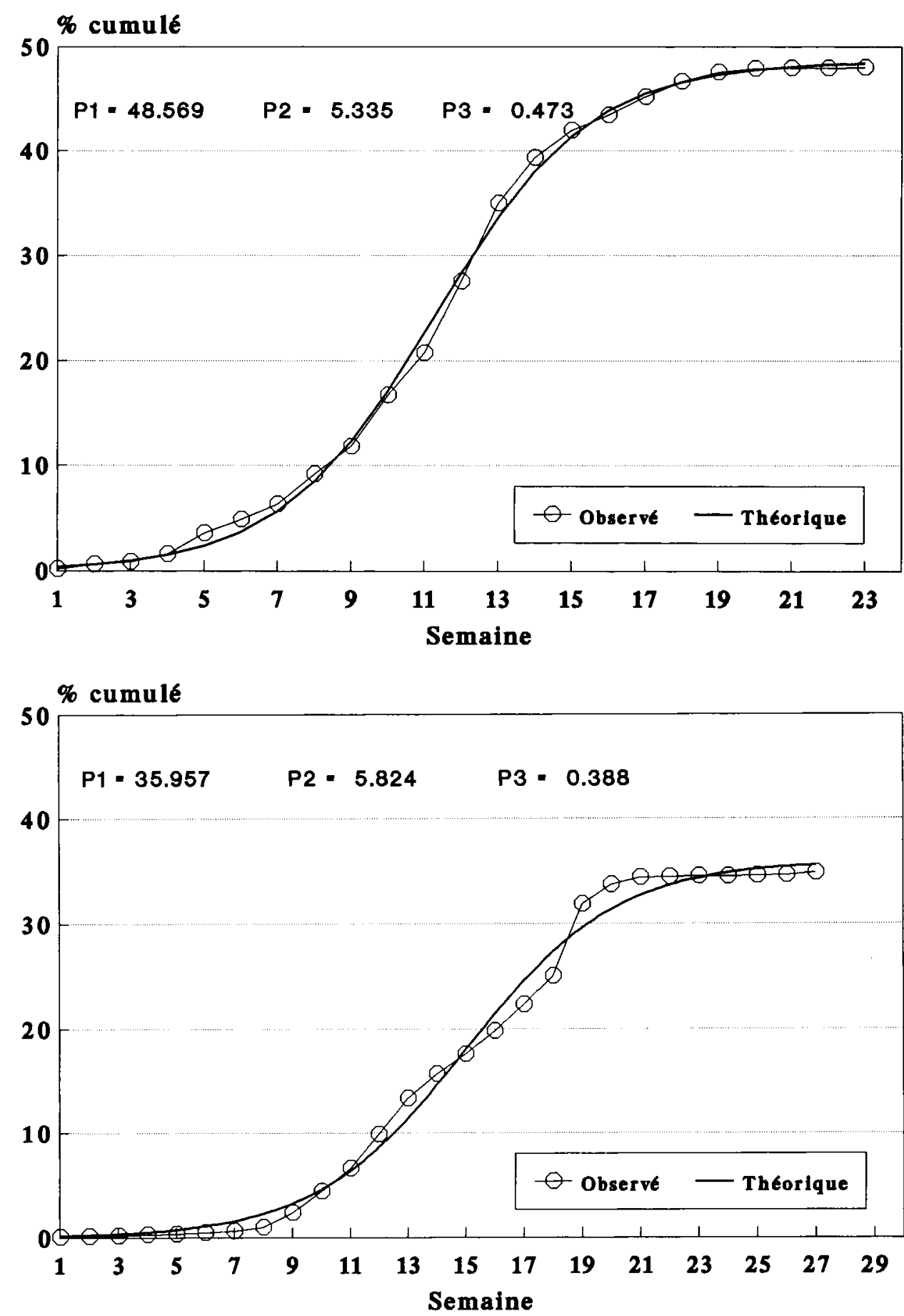

(modèle de Weibull, de Gompertz), mais les ajustements étaient moins efficaces, avec des $R^{2}$ inférieurs. Le modèle logistique permet d'expliquer le développement de l'épidémie : au début de la période de fructification, la maladie se développe suivant une courbe exponentielle, puis, dans un deuxième temps, sa progression diminue pour se stabiliser à une valeur asymptotique. L'évolution exponentielle du début de l'épidémie est classique en épidémiologie. Le changement de concavité de la courbe et la stabilisation du niveau de la maladie s'expliquent par le prélèvement, au fur et à mesure de leur apparition, des cabosses pourries, rongées ou mûres ; la progression de la maladie s'amortit donc par manque d'hôtes (cabosses), phénomène qui est accentué par les conditions de récolte sanitaire utilisées pour les comptages.

Le modèle logistique permet également d'attribuer une signification biologique aux paramètres :

- $\mathrm{P}_{1}$ : asymptote horizontale $<->$ estimation du pourcentage final de pourriture ; 


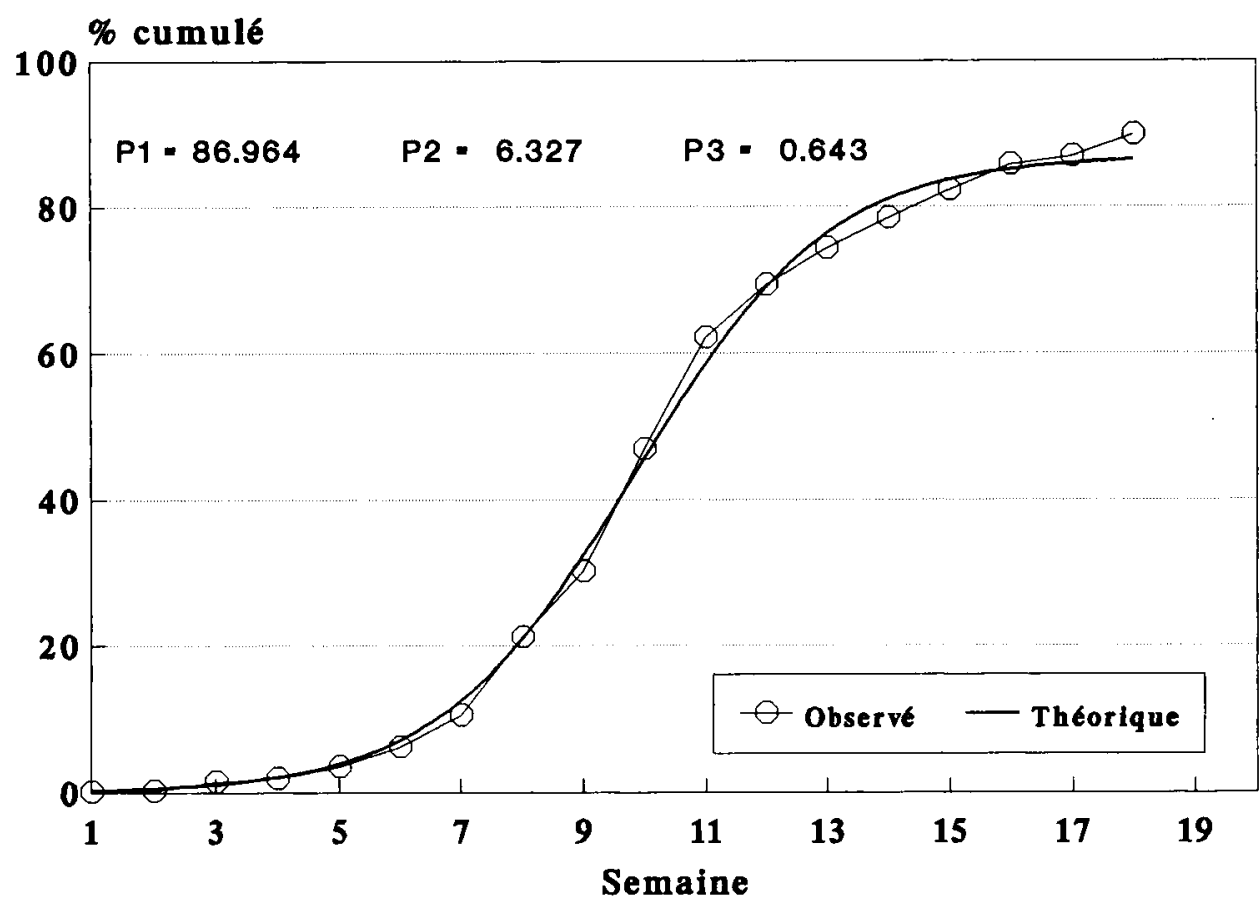

Fig 3. Ajustement du pourcentage cumulé de pourriture brune à une loi logistique (année 1990) $\left(R^{2}=0,998\right)$.
$-P_{3} \times P_{2} / 4$ : pente au point d'inflexion $\downarrow$

vitesse maximale de propagation de la maladie ;

$-P_{2} / P_{3}$ : nombre de semaines pour arriver au point d'inflexion

$\uparrow$

durée de la phase exponentielle de l'épidémie.

Une analyse diallèle sur les 2 derniers facteurs épidémiques (vitesse maximale de propagation et durée de la phase exponentielle) a été réalisée à partir des valeurs estimées sur les données de 1988 pour les différentes familles (tableau VI). Le facteur $P_{1}$ est équivalent au taux final de pourriture analysé précédemment, l'analyse de ce paramètre n'est donc pas présentée.

Un seul effet génétique significatif est détecté : celui de l'AGC pour les pentes aux points d'inflexion des courbes logistiques.
Le classement obtenu sur les AGC des pentes est globalement identique à celui obtenu avec les pourcentages finaux d'attaque de la même année ; la pente au point d'inflexion semble donc conditionner le taux d'attaque final. Le géniteur UPA 134 induit une moindre vitesse maximale de propagation de la maladie, ce qui explique en partie le meilleur comportement de ses descendants vis-à-vis de la pourriture.

\section{Effet de la répartition verticale des fruits dans les arbres}

Les analyses diallèles sur les taux de pourriture des différentes parties de l'arbre (tronc et branches) donnent des résultats comparables à ceux obtenus sur les taux de pourriture globaux : seules les AGC constituent la source de variation ayant un effet significatif. Les classements des

Tableau VI. Analyse diallèle de 2 facteurs épidémiques définis à partir des ajustements logistiques (en 1988), Valeurs des $\mathrm{F}$ (test de Fisher).

AGC

(résiduelle : $A S C$ )

Pente au point d'inflexion

Nombre de semaines pour arriver au point d'inflexion
$4,43^{*}$
$0,80 \mathrm{~ns}$
$2,49 \mathrm{~ns}$
$1,62 \mathrm{~ns}$ 
géniteurs pour leur AGC sont globalement identiques sur les 2 étages considérés et correspondent approximativement aux classements sur les taux globaux de pourriture présentés dans le tableau IV.

Cependant, les taux de pourriture observés dans les branches sont systématiquement inférieurs à ceux observés sur les troncs $(0,388<$ 0,$616 ; 0,269<0,474 ; 0,863<0,941$; respectivement pour les moyennes des 3 années successives). La répartition verticale des cabosses pourrait donc avoir une influence sur l'expression de la maladie au champ. Une analyse diallèle sur la proportion de cabosses comptabilisées dans les branches par rapport au total du nombre de fruits de l'ensemble des arbres est donc réalisée afin d'évaluer les éventuels effets génétiques de cette répartition. Pour les 3 années considérées, l'aptitude générale à la combinaison constitue la principale source de variation pour ce caractère (tableau VII). Toutefois, il est à noter qu'il existe un effet maternel significatif sur l'expression de ce caractère, ainsi qu'une ASC significative pour une année. Par ailleurs, le taux de cabosses dans les branches augmente avec le vieillissement des arbres (respectivement 0,749, 0,775, 0,793 , pour les 3 années successives). Ce fait s'explique par une croissance des branches et un développement des fleurs sur celles-ci, ainsi que par une diminution des coussinets floraux sur les troncs au cours du temps. Les classements des aptitudes générales à la combinaison estimées pour ce caractère sont présentés dans le tableau VIII.

La divergence des classements entre test d'inoculation sur cabosses et observations au champ ne peut pas être expliquée simplement par les différences de répartition des cabosses. Seul le géniteur ICS 95, dont les descendants ont une plus forte proportion de cabosses sur le tronc, manifeste une sensibilité plus importante par évaluation au champ. L'étude de l'influence de la répartition verticale des cabosses sur les taux de pourriture sera complétée par la présentation des corrélations entre différents caractères étudiés.

\section{Corrélations avec les taux de pourriture}

Les corrélations phénotypiques, génétiques et environnementales ont été calculées pour les variables : production potentielle (estimée par le nombre total de cabosses produites par croisement et par bloc), taux de cabosses dans les branches (par rapport à l'ensemble : tronc + branches) et taux de pourriture globale (sur l'ensemble des étages: tronc et branches). II s'agit principalement d'évaluer l'influence de la quantité de fruits produits et de leur répartition verticale dans les arbres sur l'expression de la maladie au champ. Ces différentes corrélations sont présentées dans le tableau IX.

Les corrélations génétiques ne sont pas stables au cours des années successives. Le taux de pourriture est corrélé génétiquement avec le nombre total de cabosses en 1988, mais cette corrélation négative s'estompe les 2 dernières années. II est possible que cette forte corrélation, observée la première année, soit due à une perte plus importante de chérelles (jeunes fruits) chez les arbres très attaqués par la pourriture. La corrélation génétique entre taux de pourriture et taux de cabosses dans les branches est négative les 2 premières années et positive la dernière ; aucune conclusion ne peut donc être tirée sur les relations génétiques entre ces 2 caractères.

En revanche, il existe une corrélation environnementale systématiquement positive entre le taux de pourriture et le nombre total de cabosses. Cette corrélation est certainement due aux infections secondaires, de cabosse à

Tableau VII. Analyse diallèle du dispositif en blocs pour le caractère de répartition verticale des cabosses. Valeurs des $F$ (test de Fisher).

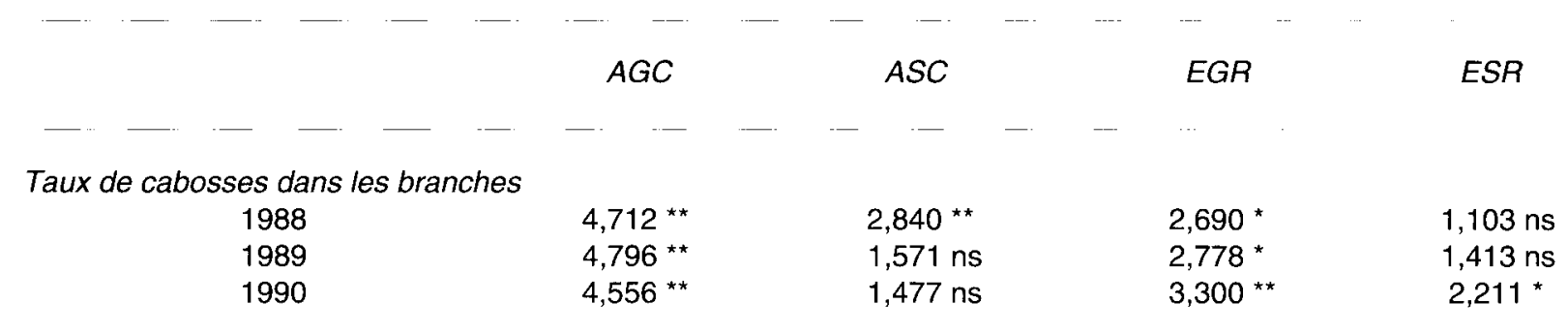

ns: non significatif à $5 \%$; ${ }^{\star}$ significatif à $5 \%$; ${ }^{\star \star}$ significatif à $1 \%$. 
Tableau VIII. Valeurs estimées des AGC par année et par géniteur $\left(\hat{g}_{j}\right)$ et tests de comparaison multiple de Newman et Keuls à $5 \%$ ( $N$ et $\mathrm{K}$ ), pour les taux de cabosses dans les branches (en \%).

Taux de cabosses dans les branches

\begin{tabular}{|c|c|c|c|c|c|c|c|c|}
\hline Clones & $\hat{g}_{i}(1988)$ & Net & & $\hat{g}_{i}(1989)$ & Net $K^{\star}$ & $\hat{g}_{i}(1990)$ & $N \in$ & $K^{*}$ \\
\hline IMC 67 & $-4,03$ & a & & $-3,45$ & a & $-1,85$ & a & \\
\hline ICS 95 & $-2,20$ & $a \quad b$ & & $-2,82$ & a & $-1,71$ & a & \\
\hline UPA 134 & 0,75 & $b$ & $\mathrm{c}$ & 0,62 & $a b$ & $-1,41$ & a & \\
\hline SNK 10 & 2,54 & & $c$ & $-1,03$ & $a \quad b$ & $-0,36$ & a & $b$ \\
\hline SNK 413 & 1,09 & $b$ & $\mathrm{c}$ & 3,81 & b & 2,44 & & $b$ \\
\hline ICS 84 & 1,85 & $b$ & $c$ & 2,86 & $b$ & 2,88 & & $\mathrm{~b}$ \\
\hline
\end{tabular}

* Les clones avec la même lettre ne sont pas différents au seuil de $5 \%$.

Tableau IX. Corrélations phénotypiques, génétiques et environnementales entre le taux de pourriture (tpour) et les variables cabtot (quantité de cabosses produites) et rapbr (taux de cabosses dans les branches).

1988 - tpour

1989 - tpour

1990 - tpour

$\begin{array}{ll}0,219 & -0,177 \\ 0,300 & -0,085 \\ 0,295 & 0,062\end{array}$

\section{Phénotypiques}

cabtot

rapbr

\section{Corrélations}

Génétiques

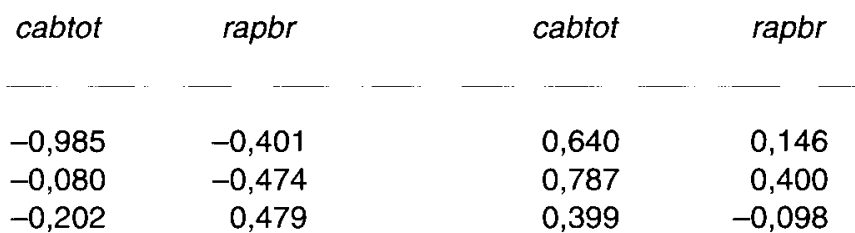

cabosse, qui sont d'autant plus importantes que la densité des fruits sur les arbres est grande. La corrélation environnementale entre le taux de pourriture et le taux de cabosses dans les branches n'est pas stable au cours des années successives.

\section{DISCUSSION ET CONCLUSIONS}

L'étude génétique de la réaction à la pourriture brune dans la parcelle diallèle de Barombi-Kang a confirmé les résultats obtenus par Despréaux et al (1989), dans des conditions naturelles de niveaux d'infection plus élevés.

Les différentes analyses mettent en évidence la supériorité du géniteur UPA 134, avec de bonnes AGC pour les caractères considérés. Ce clone permet d'obtenir des hybrides productifs, présentant de faibles taux d'attaque ; ce géniteur devrait donc être plus largement utilisé dans les champs semenciers du Cameroun.
Le meilleur comportement des descendants du clone UPA 134 est dû, en partie, à une plus faible vitesse de propagation de l'épidémie, estimée par le modèle logistique.

Lors de travaux antérieurs (Berry et Cilas, 1994), une assez bonne relation entre AGC et valeurs propres des clones pour le taux de pourriture observé en champ avait été mise en évidence ; le clone UPA 134 se situait parmi ceux présentant les plus faibles proportions de cabosses atteintes. Toutefois, ce clone a été identifié comme sensible lors d'inoculations artificielles sur cabosses (Blaha et Lotodé, 1976) ; il est probable que ce mode d'évaluation, qui reflète le niveau de résistance intrinsèque de la cabosse, ne permette pas de révéler tous les facteurs ayant un rôle dans l'évolution de la maladie au champ, comme dans le cas de résistance partielle.

La charge des arbres en cabosses et la répartition des fruits dans les arbres ne permettent pas d'expliquer les divergences de classement entre 
le test d'inoculation sur cabosses et les observations de la maladie en champ.

Des données sur la durée des cycles de fructification, collectées à la station IRA de Nkoemvone (Cameroun), permettent de formuler une hypothèse pour expliquer cette divergence (cf Annexe). En effet, alors que le début de la floraison des différents clones est simultané, en relation étroite avec la pluviométrie, la récolte des cabosses est étalée dans le temps. La comparaison de différents clones pour la durée du cycle de fructification, dans les conditions climatiques du Sud Cameroun, a permis de mettre en évidence des différences importantes et d'apporter une explication de la divergence entre les diverses méthodes d'évaluation du matériel végétal pour leur réaction au Phytophthora. Dans le tableau présenté en annexe, différents clones sont comparés pour la durée de leur cycle de fructification, exprimée en nombre de jours qui s'écoulent entre la nouaison et la récolte de la cabosse mûre. Ce caractère sépare très bien les différents groupes de cacaoyer (ICS, SNK et UPA).

Les ICS ont des cycles de fructification longs (environ $200 \mathrm{j}$ ) ; l'exposition de leurs cabosses au pathogène, en condition naturelle d'épidémie, dure donc plus longtemps. Les 2 ICS étudiés ici apparaissent effectivement plus sensibles en champ que lors des tests d'inoculation artificielle sur cabosses. En revanche, les cabosses du clone UPA 134 restent moins longtemps dans les arbres, et sont donc moins longtemps exposées à la maladie. Ce clone est en effet évalué comme l'un des plus résistants au champ, alors qu'il avait été jugé sensible lors des tests d'inoculation. Les clones SNK, ayant des longueurs de cycle intermédiaires, ont des sensibilités (estimées) comparables pour les différentes méthodes d'évaluation. La longueur de la période de développement des cabosses permet donc d'expliquer en partie ces différences de comportement : les clones à cycle de fructification long apparaissent plus sensibles au champ que lors d'inoculations artificielles car l'exposition de leurs cabosses au pathogène dure en moyenne plus longtemps. Le décalage du cycle de fructification par rapport au cycle du champignon ainsi que la longueur de ce cycle avaient déjà été suspectés d'intervenir sur le développement de la maladie en champ (Muller, 1974).

L'étude des cycles de fructification des génotypes retenus dans les schémas de sélection apparaît donc comme une étape nécessaire. Ce caractère, complémentaire du facteur de résis- tance intrinsèque observable lors d'inoculations artificielles, doit être pris en compte dans le cadre de la mise au point d'une méthode de lutte intégrée. En effet, du matériel végétal à cycle de fructification plus court nécessiterait vraisemblablement une protection par applications de fongicides moins longue, donc moins coûteuse.

Dans cet essai diallèle, une importante variabilité intra-famille est observée ; celle-ci est due à la structure hétérozygote des géniteurs. À l'intérieur des familles les moins sensibles au champ, une sélection d'arbres bons producteurs et présentant une faible incidence de la maladie est envisagée, selon une sélection combinée «individus/famille» sur index.

\section{REMERCIEMENTS}

Les auteurs remercient $M \mathrm{~J}$ Ayuk Takem, directeur de I'IRA, M J Awemo, chef de la station de Barombi-Kang, M A Kadjé, chef de la station de Nkoemvone, MM J Kitio et Mbar-Ndjié, chefs des équipes d'observateurs "cacao" des stations de Barombi-Kang et Nkoemvone, et toute l'équipe de saisie informatique de la station de Nkolbisson, qui ont permis la réalisation de ces travaux.

\section{ANNEXE}

Comparaison de clones pour la durée du cycle de fructification.

$\begin{array}{ccc}\text { Clones } & \begin{array}{l}\text { Moyennes } \\ \text { (en jours) }\end{array} & \begin{array}{c}\text { Groupes homogènes } \\ \text { (Bonferroni 5\%) }\end{array}\end{array}$

\begin{tabular}{|c|c|c|c|c|c|c|}
\hline ICS 61 & 209,5 & a & & & & \\
\hline ICS 43 & 206,0 & $\mathrm{a}$ & & & & \\
\hline ICS 60 & 202,2 & a & $b$ & & & \\
\hline ICS 46 & 201,1 & a & $b$ & & & \\
\hline ICS 39 & 199,8 & a & $b$ & & & \\
\hline ICS 40 & 197,8 & & b & & & \\
\hline ICS 16 & 197,4 & & b & & & \\
\hline ICS 84 & 195,0 & & $b$ & & & \\
\hline SNK109 & 187,3 & & & c & & \\
\hline SNK 30 & 187,2 & & & c & & \\
\hline SNK 460 & 183,2 & & & c & $d$ & \\
\hline SNK 10 & 181,6 & & & c & $d$ & \\
\hline SNK 48 & 177,2 & & & & $d$ & \\
\hline SNK 416 & 175,4 & & & & d & e \\
\hline SNK 16 & 175,2 & & & & $d$ & e \\
\hline SNK 111 & 174,3 & & & & d & e \\
\hline SNK 13 & 172,4 & & & & d & e \\
\hline UPA 134 & 168,3 & & & & & e \\
\hline UPA 143 & 163,1 & & & & & \\
\hline
\end{tabular}




\section{RÉFÉRENCES}

Berry D, Cilas C (1994) Étude du comportement d'une parcelle diallèle $6 \times 6$ vis à vis de la pourriture brune des cabosses du cacaoyer due à Phytophthora spp au Cameroun. In : Proc 11th Int Cocoa Res Conf, Yamoussoukro, Côte d'Ivoire (Stephen Austin and sons, eds) (sous presse)

Blaha G (1974) Methods of testing for resistance. In : Phytophthora Disease of Cocoa (PH Gregory, ed), Longman, Londres, Royaume-Uni, 259-268

Blaha G, Lotodé R (1976) Un caractère primordial de sélection du cacaoyer au Cameroun : la résistance à la pourriture brune des cabosses. Café Cacao Thé 20, 97-116

Blaha G, Lotodé R (1977) Contribution à la connaissance des modalités de la transmission héréditaire de la résistance du cacaoyer à la pourriture des cabosses (Phytophthora palmivora) au Cameroun. Café Cacao Thé 21, 179-196

Brasier CM, Griffin MJ (1979) Taxonomy of Phytophthora palmivora on cocoa. Trans $\mathrm{Br} \mathrm{Mycol}$ Soc 72, 111-143

Dattée $Y$ (1972) Analyse quantitative de l'auto- et de l'interfertilité chez quelques familles de luzerne. Ann Amélior Plant 22, 5-21

Despréaux D, Cambrony D, Clément D, Partiot $M$ (1988) Étude de la pourriture brune des cabosses du cacaoyer au Cameroun : définition de nouvelles méthodes de lutte. In : Proc 10th Int Cocoa Res Conf, Santo Domingo, République dominicaine (Stephen Austin and sons, eds), 407-412

Despréaux D, Clément D, Partiot M (1989) La pourriture brune des cabosses du cacaoyer au Cameroun : mise en évidence d'un caractère de résistance au champ. agronomie 9, 683-691

Griffing B (1956) Concept of general and specific combining ability in relation to diallel systems. Aust $J$ Biol Sci 9, 463-473

Hartley HO (1961) The modified Gauss-Newton method for the fitting of non-linear regression functions by least squares. Technometrics 3, 269280

Lass RA (1985) Phytophthora pod rot (often called "black pod"). In : Cocoa (GAR Wood, RA Lass, eds), Longman, Londres, Royaume-Uni, 267-282

Muller RA (1974) Integrated control methods. In : Phytophthora Disease of Cocoa (PH Gregory, ed), Longman, Londres, Royaume-Uni, 259-268

Nyasse S (1992) Structure d'une population de Phytophthora $s p$ des cacaoyères camerounaises atteintes de pourriture brune. DRU sciences agronomiques, Inst nat polytech de Toulouse, France

Partiot M (1975) La résistance horizontale du cacaoyer au Phytophthora species. Café Cacao Thé 19, 123130

Tan GY, Tan WK (1990) Additive inheritance of resistance to pod rot caused by Phytophthora palmivora in cocoa. Theor Appl Genet 80, 258-264

Tarjot M (1969) Étude de la résistance des cacaoyers à la pourriture brune des cabosses due à Phytophthora palmivora (Butl) Butl en Côte d'Ivoire. $3^{\mathrm{e}}$ partie : inoculations expérimentales sur le terrain. Café Cacao Thé 13, 297-309

Thorold CA (1953) The control of black pod disease of cocoa in the western region of Nigeria. Rep Cocoa Cont, Londres, 108-115 